\title{
A UNIVERSAL ICT DEVICE CONTROLler FOR THE Visually Challenged
}

\author{
Suraj $\mathrm{R}^{1}$, Dilraj $\mathrm{N}^{2}$ and Sethuraman $\mathrm{Rao}^{3}$ \\ ${ }^{1-3}$ Amrita Center for Wireless Networks and Applications, \\ AMRITA Vishwa Vidyapeetham, Amrita University \\ Amritapuri, Kollam, India.
}

\begin{abstract}
With today's modern lifestyle, ICT devices that were once considered as luxuries have turned into necessities. One of the main problems associated with these ICT devices is that they all come with separate remote controllers. All these remote controllers have got different buttons which are designed in their own customized way. So there is a lack of commonality or interoperability between different devices or between different vendors. Now, this becomes a major problem when the visually challenged people need to use these devices. In this paper, we present a novel approach which acts as a universal intelligent remote controller for all the electronic devices and which is extremely user-friendly to the visually challenged. It uses two transceivers- one at the ICT device end which is used to communicate directly with the ICT device and the other is the user end device i.e. the Smart phone.
\end{abstract}

\section{KEYWORDS}

Android application; Visually impaired; ICT devices.

\section{INTRODUCTION}

I-Controller is an Intelligent Universal Remote Controller for use by the visually challenged and other people. In our 21 st century lifestyle, yesterday's luxuries have become today's necessities. In today's world there are some devices and gadgets which we can't live without, for example TV, refrigerator, washing machines, coffee makers, AC's etc.

One of the main problems associated with ICT electronic devices is that they all are using separate remote controllers for their controlling part. All these remote controllers have got different buttons which are arranged in their own customized way. So there is no commonality or interoperability between different devices or between different vendors. The technological revolution failed to touch this area since people seem to be comfortable with this scenario.

But this becomes a major problem when it comes to special-needs people like the visually challenged. So in this paper we propose a new device which is supposed to be a universal intelligent remote controller for all the electronic devices which are currently available in the market or yet to be and which will cater to the needs of the special groups such as the visually challenged. It uses two transceivers - one at the ICT device end which is used to communicate directly with the device and the other at the user end device i.e. the Smart phone. The main attraction of this system is the two way communication capability of the controller with the ICT device.

DOI: $10.5121 /$ ijci.2015.4216 
International Journal on Cybernetics \& Informatics (IJCI) Vol. 4, No. 2, April 2015

The remaining part of this paper is organized as follows: section II describes related work in the area of blind navigation in indoor and outdoor environment. Detailed system architecture is illustrated in section III. Section IV discusses the implementation details. Section V deals with simulation results and Section VI concludes with future research scope and expansion.

\section{RELATED WORK}

Way back in 1970's controllers of ICT devices were remotely manipulated using ultrasonic tones. Later it evolved to infrared that we use in today's remote controls. The concept of universal remote controls is not something new to the industry but the problem is there is no universality in these controlling methods.

The main problem with ICT device controllers is that there are no practical systems which are currently available which serves the purpose of ICT device control for the visually challenged.

An advancement in technology is TeslaTouch [1][2][3] which has been developed by Disney Research, Pittsburgh; Universite` Paris-Sud; Human-Computer Interaction Institute, Carnegie Mellon University. Teslatouch is an innovation in the touch interface of future mobile phones. In this technology the user will eventually get a feel of what they are touching.

TeslaTouch is an interactive touch interface that is compatible with current day display interfacing technologies. TeslaTouch has got a 3 layer display module consisting of a micro touch panel, transparent electrode and an insulator layer. When a signal is passed to the transparent electrode display, it produces electro-vibrations at the instant when the finger touches the display (Capacitive display was used for testing). So like the Braille scripting the user will get a perception of the letters that are being displayed. As of now TeslaTouch technology can only perceive letters effectively. This can also be a future enhancement of the proposed project. If the Smart phone companies adopt this technology then the visually challenged people can more intuitively interact with the mobile phone for controlling along with the talking signage provided by mobile OS's such as Android and iOS.

Smart phones have increased the popularity of Bluetooth as a communication medium manifold. The programmers guide called Bluetooth for programmers [4]

describes how to edit the Bluetooth stack programmatically. One option is to use friendly names to bypass the pairing authentication. However, the problem with this type of pairing is the time required for accepting the connection and the high energy cost involved.

For eliminating all the disadvantages of conventional Bluetooth devices a newer version, Bluetooth 4.0[5] or popularly known as Bluetooth Smart has been launched. It eliminates all the shortcomings of conventional Bluetooth, with internal support for automatic pairing. It is fast and highly energy efficient. The problem as far as I-Controller is concerned is that this technology is being incorporated in new Smart phones only. So most of the Smart phones in use today will not support Bluetooth Smart technology.

Smart TVs[6] are quite common these days. But the main problem till recently was the absence of a common development platform. Thus the Smart TV alliance came into being. They agreed upon some common firmware standards so that the development cost can be reduced to the maximum extent. This brought all the smart TV manufacturers and application vendors under a single alliance. But their work addressed the Smart TV ICT devices only.

Another patented work[7] in this field is transmitting data via any RF medium whereas the origin and destination will be IR itself. 
Also there are a lot of remote laptop/PC controlling mobile apps [8] that are available in the market. The problem with these systems is that a server must be hosted at the laptop end. So practically it's not possible to implement such a system in other non PC based ICT devices.

Tiresias[9] seems to be the only provider which researches on making ICT devices accessible to the visually challenged. But the feasibility issues due to mobility, cost, bulkiness, etc., make it almost out of reach for majority of the people.

Fin[10] is a smart wearable ring, which can be used to control devices via gestures. The four fingers in hand are separated as three sectors each and each sector is allocated to control a particular parameter of a device. The thumb finger can be used to select any of these sectors using gesture. The problem of this technology is that the form factor or the number of devices and controls assigned are limited to the sectors in each finger.

\section{ARCHITECTURE AND DESIGN}

The proposed system in Figure 1 consists of two modules:

\subsection{Smart ICT Controller Module}

The Smart ICT Controller is the heart of this system. It has the ability to decode the Bluetooth signals to corresponding IR patterns.

Whenever the Smart module establishes a connection with the I-Controller application (which is the Android remote control part explained in Section B), it can decode and read any data coming from the I-Controller application so that the ICT device can be controlled accordingly.

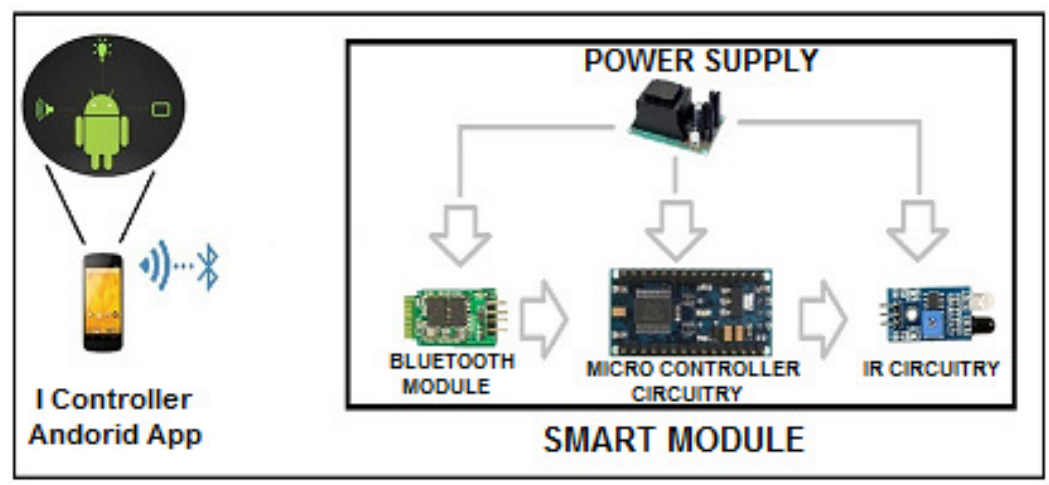

Figure 1. Architecture

\subsection{I-Controller}

I-Controller is a proposed Android Application which can be installed in any of the Smart phones running Android based OS. The application will detect the devices which are within the range of the Smartphone. The Visually challenged user can connect to any particular device with the aid of Android's talkback facility (Talkback is an inbuilt facility in Android based Smartphones. User can enable it from accessibility option in the settings of their Smartphones). Once the connection is established, the I-Controller can decode the ID of incoming ICT device, present an appropriate GUI (I-Controller has some pre-coded GUI's compatible for commonly available ICT devices) 
for controlling the device and talkback the features (pre-coded in I-Controller) of the device being controlled.

Whenever the android device comes in the vicinity of a particular device/devices it will show up all the devices within range. With the help of talkback facility, the visually challenged can hear which all devices are listed and where to touch in order to select the particular device.

Once the device is selected, the user will be getting a brief note regarding the details of the ICT device to which they have connected and the controls of the same (Figure 2).

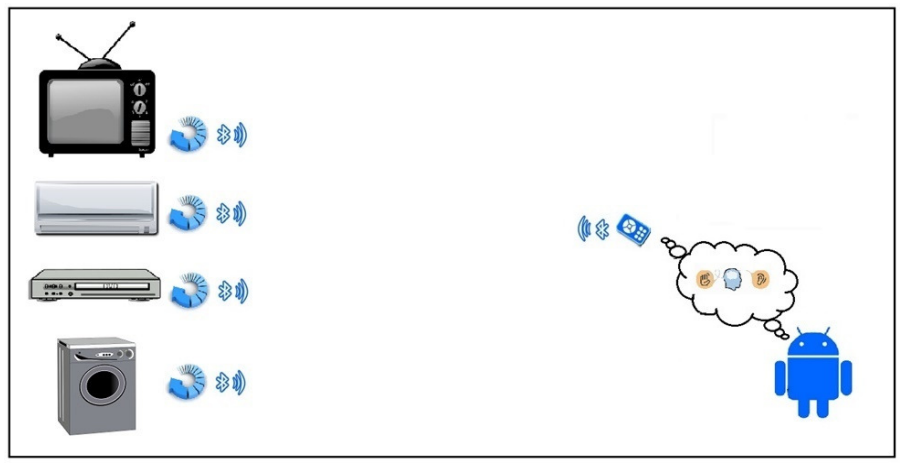

Figure 2. I-Controller

\section{IMPLEMENTATION}

The Smart ICT Controller Module will be installed in the vicinity of each ICT device at home. The proposed Smart module is external one. The Smart module is placed in the vicinity of the IR receiver of the ICT device. Since the Smart module can be manufactured economically, buying a separate Smart module for every single ICT device will not cost much.

\subsection{Smart module Components}

\subsubsection{Power supply unit}

A power supply unit is employed to power up the PIC and Bluetooth device.

\subsubsection{Bluetooth Module}

A Bluetooth module is installed to receive the incoming signals from the I-Controller Android application. The Bluetooth module used is Blue Link Silver Bluetooth Module. The module is shown in the Figure 1.

\subsubsection{Circuitry}

The main logic implemented by the microcontroller circuitry is to send device ID once a paired device is found near its vicinity. The controller is equipped with the logic to decode the Bluetooth signals and to convert it to infrared logic. It implements the important function of mapping devices. It contains the important information regarding the ICT device which it is being connected to. Most importantly it has the codes to decode the incoming data so that it can process the data and transform it to corresponding IR patterns. 


\subsubsection{IR Circuitry}

The output pattern produced by the Pulse Width Modulator is fed to the IR circuitry and it generates the required IR signal. Transistor as a switch is being used here to boost the current levels. A $38 \mathrm{KHz}$ IR transmitter is being used as the IR transmitter which suits most devices out in the market.

\subsection{Setup Configuration}

\subsubsection{One to one Smart module}

In this setup a single Smart module is deployed for each ICT device. So each Smart module will be communicating only with its own ICT device. This type of Smart module is so easy to setup. Moreover this type of Smart modules have pre-locked program memory. It won't support OTG editing of firmware once it is deployed. Just connect supply to the Smart module and switch it on.

\subsubsection{One to many Smart module}

In this setup single Smart module can serve multiple ICT devices. So its placement in the room during installation is an important parameter. This type of Smart modules can

\subsubsection{Internal Smart module}

This setup is a future scenario where the Smart module comes inbuilt in the ICT device by the manufacturer. That's a future vision of this project.

When the visually challenged person moves around with the Smart phone whose inbuilt talkback feature is enabled, the I-Controller applications on the Smart phone will detect the available Bluetooth equipped devices. The visually challenged can get to know what all devices are displayed with the help of a finger touch. Upon a finger touch, talkback option will speak out the name of the device which is touched. Upon double tap the device will get selected.

Those devices other than ICT devices will not establish a successful connection, (e.g. devices like Smart phones) even if they are selected from the list. This is because of a unique ID set for the proposed I-Controller application. Because of that the I-Controller will not establish a connection with any device other than ICT device.

Once the connection is established the I-Controller will talkback a brief description about the device to which it is currently connected. Along with that, a layout of the controls of the ICT device will be displayed as a GUI. The visually challenged person can access this GUI using the talkback facility.

\subsection{Softwares used}

\subsubsection{Eclipse IDE with Android Development Tools}

Eclipse Android Development tools are being used for the development of the Android Smartphone application. The application is developed in such a way that it supports all android Smartphones from Gingerbird to Lolipop. All the SDK for all the versions are therefore installed in the IDE. 


\subsubsection{CCS- PICC}

CCS-PICC compiler is a user friendly compiler which has many inbuilt libraries and utilities. It is being used for developing embedded $\mathrm{C}$ codes.

\subsubsection{KiCad}

KiCad is being used for designing the layout for making the final PCB.

For testing purposes we used PIC development board. With the help of CCS PICC compiler we could even test serial data. The CCS compiler provides a Graphical User Interface for sending any format of serial data.

The PIC development board has got both $5 \mathrm{v}$ and $12 \mathrm{v}$ supply out slots. This helps in directly powering up external Bluelink Bluetooth module.

In the initial phases before developing the Smart module unit, we were able to test different formats of data like bytes or character strings. It yielded very good results which helped in altering the Android application code and thereby formatting the real data which needs to be sent from the Smart module end.

Whenever the Bluetooth module and Android Smatphone establishes a connection they internally agree on a common baudrate that helped to ease that part. But during testing times the baudrate in the GUI of serial data interface need to be manually set to 11500 .
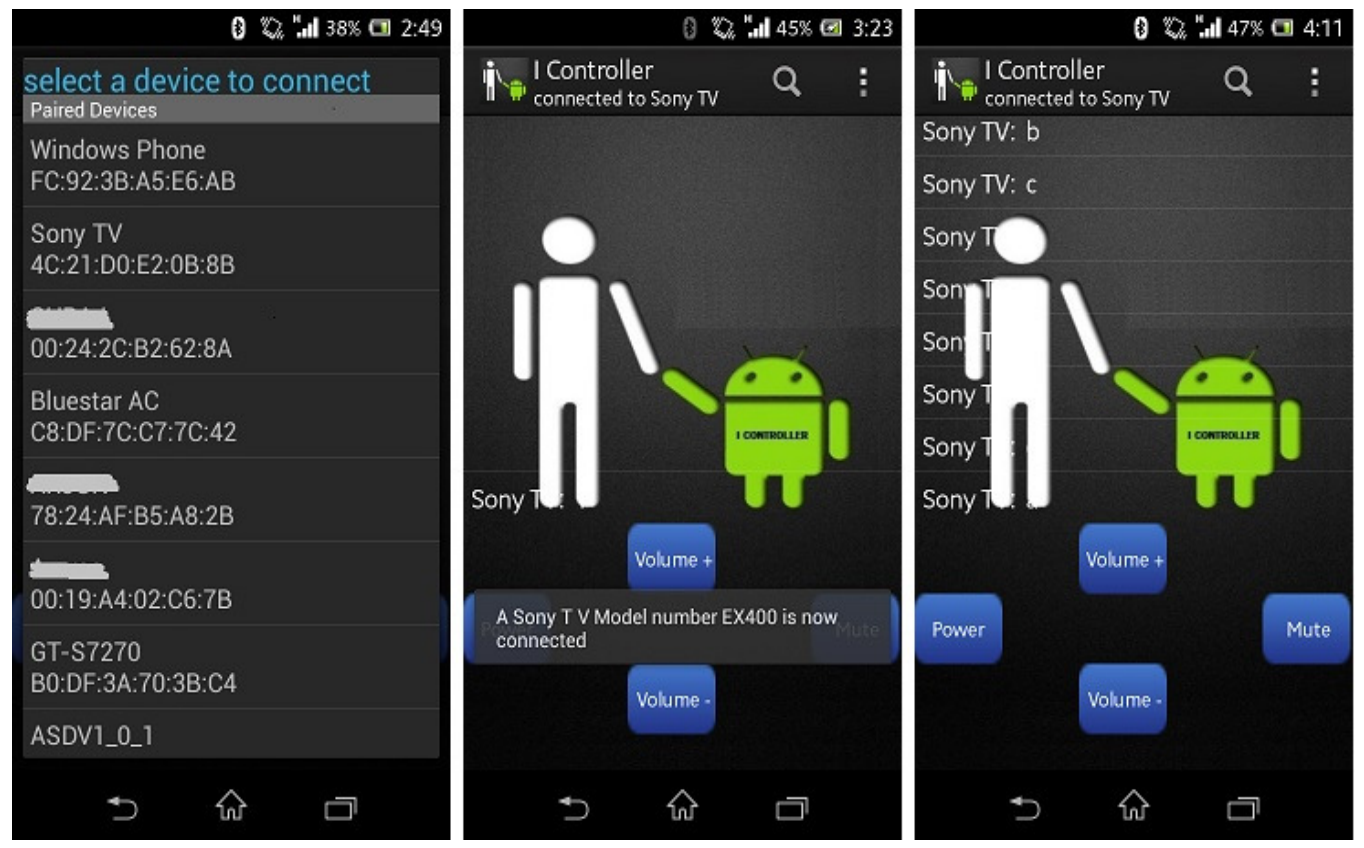

Figure 3. Android Application

Figure 3 shows the Screenshots of the I-Controller application. First Screenshot in Figure 3 shows the ICT devices it has detected within its range. Talkback facility in Android has the ability to speak out the listed devices and helps the visually challenged to select a particular ICT device. 
Once a device is selected, the I-Controller application receives a brief description message of the ICT device from the ICT device end Smart controller as shown in the second screenshot in Figure 3.

After the communication with the device is established, the I-Controller application receives the details of the controls of the selected device from the Smart controller module. These details are shown as a GUI screen by the I-Controller application. This is shown by the third screenshot in Figure 3

When the smart module of the selected device communicates with I-Controller it sends unique bytes or combinations to reveal information about itself. The I-Controller has the intelligence to map the received bytes to particular pre-stored information about the controls on the device.

The user can select any device like Sony TV listed in Figure 3 and accordingly the I-Controller brings in a dedicated GUI to control that particular ICT device. The control persists as long as the Smartphone stays within the range of Smart module. The main advantage of this range limitation is that the devices will be listed only when the user is within a particular vicinity of smart module.

It also acts as an ICT equipment detector too. So wherever the visually challenged moves inside a house they will be informed about the Smart module equipped ICT devices, then it's the choice of the user whether to select a particular to ICT device or not. The whole system is programmed in such a way that always an acknowledgment is provided for each communication happening between the Smartphone and the android device.

\section{FLOWCHART}

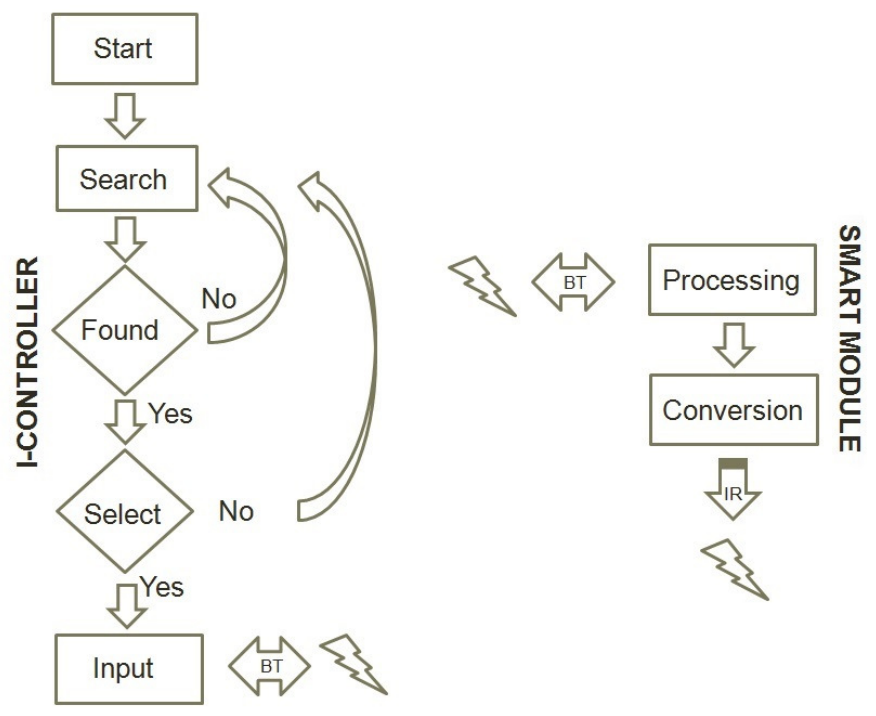

Figure 4. Flowchart

The flow of the whole system (Figure 4) is as follows. 


\subsection{I-Controlle}

- Start the application.

- Initiate a search.

- $\quad$ Lists the devices.

- $\quad$ Select a device if listed or initiate a new search.

- $\quad$ Now after establishing a successful connection the user can give inputs.

- That inputs are being transmitted via inbuilt Smartphone Bluetooth.

\subsection{Smartmodule}

- $\quad$ Receives the signals transmitted wirelessly via Bluelink Bluetooth module.

Uses an embedded system (Smart module) to decode the incoming signals and transform it to IR patters which is again transmitted to the ICT device.

\section{EXPERIMENTAL RESULTS}

The paper is intended to demonstrate a universal ICT device controller for the visually challenged. In an experimental setup the system was able to communicate with multiple ICT devices with limited controlling functionalities. The Figure 5 shows the initial experimental setup with the development board. It consists of screenshots of the I-Controller; Development board based initial Smart module testing and IR decoding using a DSO. The DSO is being used for finding out the signalling patterns of different devices using their IR remote controllers.

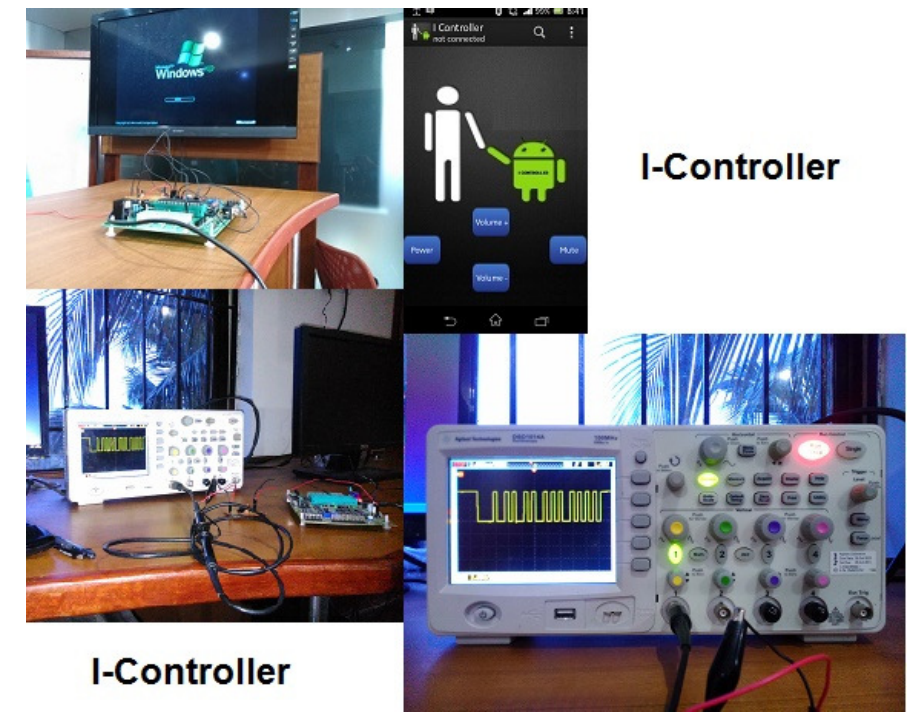

Figure 5. Testing

As discussed in implementation section, a prototype has been made and tested by controlling two devices. A Sony TV's Power ON/OFF, Volume Up, Volume Down and Mute facilities has been successfully tested. A second device, i.e, a Bluestar AC's Power ON/OFF functionality has also been tested. The Smart module prototype is being shown in the Figure 6. It shows the Smart module box and its adapter. 


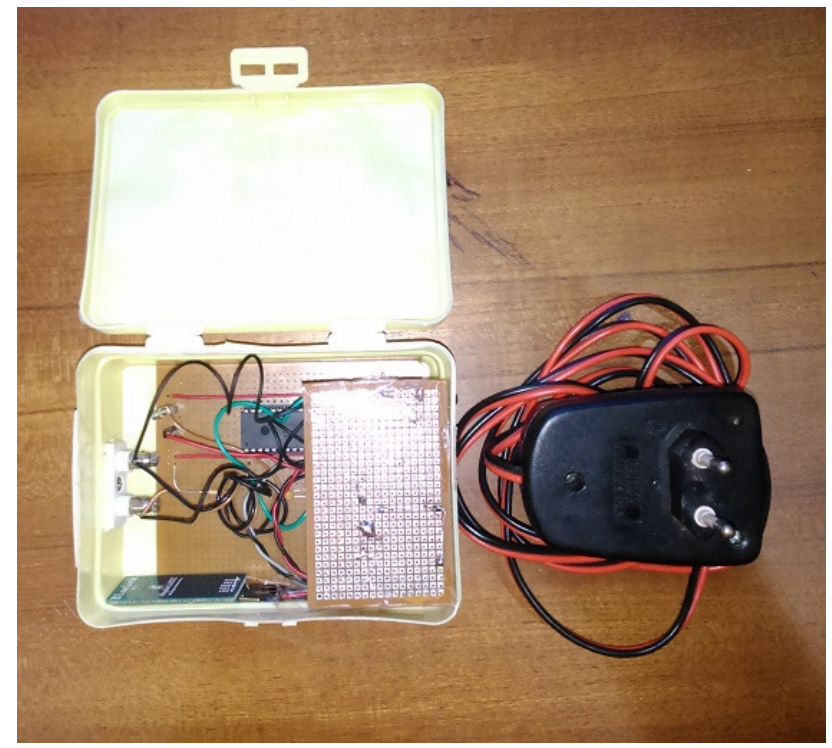

Figure 6. Smartmodule

\section{LIMITATIONS}

This system assumes the Smartphones are pre-paired. That is for all devices a onetime pairing is needed, after which the Smartphone can establish connection with any of the devices at anytime. Also it assumes there is no difficulty for the visually challenged with the inbuilt talkback functionality of android Smartphones.

\section{CONCLUSION AND FUTURE WORK}

Compared to any of the existing assistive technologies which are available now for the visually challenged, this new proposed work stands out for the uniqueness of its approach to assisting the visually challenged to access the ICT device controls. In home assistance for the visually challenged is barely touched area of research. The difficulties for those people to access or control ICT devices therefore a serious issue. With the development of this project we aim to make the ICT devices more and more accessible to them. The scope of expansion of this project is vast and therefore in a near future a complete dependency can be achieved for the visually challenged to control any electronic device in their home or a private building just by using their Smartphones.

A number of future enhancements are possible for this project. For instance, we can reduce the overhead of the I-Controller by deploying dynamic GUIs based on any light weight protocol exchange with the smart module. Through that mechanism, the layout of the ICT device can be sent at real time to the user and displayed in the GUI

of the I-Controller. This will make the I-Controller application really light-weight making it to store almost no data in its backend. Also, when Teslatouch equipped Smart phones become popular in the future, the proposed system can be an asset for the visually challenged. 


\section{REFERENCES}

[1] Olivier Bau, Ivan Poupyrev, Ali Israr, Chris Harrison ," TeslaTouch: Electrovibration for Touch Surfaces", Disney Research, Pittsburgh; Universite' Paris-Sud; Human -Computer Interaction Institute, Carnegie Mellon University, 2010

[2] Collins, "Tactile television - mechanical and electrical image projection", IEEE Transactions on Man-Machine Systems, 1970

[3] Luk. J, J. Pasquero, S. Little, K. MacLean, V. Levesque, V. Hayward, "A role for haptics in mobile interaction: Initial design using a handheld tactile display prototype.”, 2006: ACM. pp. 171-180

[4] Albert Huang, Larry Rudolph, Bluetooth for Programmers.Available: people.csail.mit.edu/rudolph/Teaching/Articles/BTBook.pdf

[5] Bluetooth Smart Technology: Powering the Internet of Things, http://www.bluetooth.com/Pages/Bluetooth-Smart.aspx

[6] Smart TV alliance press conference, "CES 2013", 2013

[7] Remote operation of local or distant infrared-controllable and non-infrared- controllable devices. US Patent: US20060210278.

[8] Win-Remote Control. Available: http://remote-controlcollection.com/download/server/windows/

[9] Making ICT Accessible. Available: http://www.tiresias.org/announcement.htm

[10] Fin : Wearable Ring Make your Palm as Numeric Keypad. Available: http://www.finrobotics.com/

\section{AUTHORS}

Suraj R received his B.Tech in Electronics and Communication Engineering from Mahatma Gandhi University, Kerala, India. He is experienced in Security based Embedded Systems, Android programming and Network Security. His fields of interest are Embedded Systems, Infotainment Systems and Cyber Security. He is currently pursuing M.Tech in Wireless Networks and Applications at Amrita School of Engineering, Amritapuri, Kollam, Kerala. His ongoing projects are Assistive technologies for Visually Challenged and in home/office remote automation and Android application for Women Safety.

Sethuraman Rao has more than 20 years of progressive technical and management experience in high-tech product development involving hardware systems, embedded software systems $(\mathrm{C} / \mathrm{C}++)$ and applications for Enterprise switching, Carrier Ethernet, IP/MPLS, ATM networking technologies and enterprise Java applications. Over 12 years of first and second level engineering management experience including management of offshore teams and outsourced projects. Solid SDLC experience bringing multiple carrierclass systems and applications to market.

Dilraj N. currently a Research Associate at the Amrita Center for Wireless Networks \& Applications, Amritapuri Campus. He is currently involved in an ongoing innovative project named Amrita Spandhanam., which has the capability of monitoring cardiac arrests and associated emergencies remotely. He has dual Masters degree, M.Tech in Wireless Networks and Applications and M.Sc in Electronics.
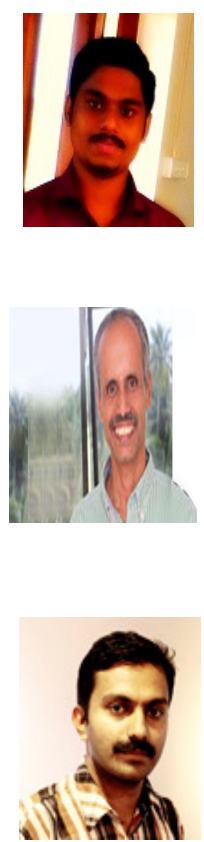